complications in gastric cancer patients undergoing surgery. The preoperative CCR showed a good predictive ability for short-term postoperative complications, with an optimal cut-off value of 7.117. Patients with low CCR had a higher incidence of overall complications $(\mathrm{P}<0.001)$, including mild complications $(\mathrm{P}<0.001)$ and major complications $(\mathrm{P}<0.001)$.

Conclusions The preoperative CCR was identified as a reliable nutrition and sarcopenia assessment tool for predicting shortterm prognosis for patients with gastric cancer after surgery.

\section{IDDF2020-ABS-0159 A NEW DIAGNOSTIC INDEX OF SKELETAL MUSCLE MASS FOR PREDICTING SHORT- TERM POSTOPERATIVE COMPLICATIONS IN CROHN'S DISEASE}

Xiaolong Ge, Linna Ye, Qian Cao, Wei Zhou. Sir Run Run Shaw Hospital, School of Medicine, Zhejiang University, China

\subsection{6/gutjnl-2020-IDDF.101}

Background Skeletal muscle depletion has been suggested to affect postoperative complications in surgical patients. The creatinine-to-cystatin $\mathrm{C}$ ratio (CCR) is demonstrated as a new marker of muscle mass. The present study investigated the role of CCR in predicting postoperative complications in Crohn's disease (CD).

Methods This cohort study included 344 CD patients undergoing bowel resection from January 2016 to June 2019 at the Inflammatory Bowel Disease Center, Sir Run Run Shaw Hospital. Univariate and multivariate analyses were performed to identify risk factors for postoperative complications in CD. Receiver operating characteristic (ROC) curves were developed to examine the cutoff value of predictors in postoperative complications.

Results Among all the patients, 120 (34.9\%) had postoperative complications and $224(65.1 \%)$ recovered uneventfully. Preoperative albumin, C-reactive protein, white blood cell, creatinine, cystatin C, CCR, operative time, surgical history, laparoscopy, estimated blood loss, azathioprine and corticosteroids usage, and enteral nutrition were demonstrated to predict postoperative complications in CD (all $\mathrm{p}$ value $<0.05$ ). Additionally, higher white blood cell $(p=0.008)$, lower creatinine $(p<0.001)$, higher cystatin $C(p=0.002)$, lower CCR $(p=0.002)$, and enteral nutrition $(p=0.001)$ were identified to be independent risk factors in CD patients. CCR was identified to be with the highest area under the curve compared with other predictors, and the cutoff value was 7.804. Patients with a CCR $<7.804$ experienced more postoperative complications, including surgical site infections $(\mathrm{p}<0.001)$.

Conclusions CCR was a simple and useful predictor as evaluating skeletal muscle mass to identify patients with a high incidence of postoperative complications in CD.

\section{IDDF2020-ABS-0160 PREVALENCE AND IMPACT OF FRAILTY AMONG INFLAMMATORY BOWEL DISEASE OUTPATIENTS}

Nikola Mitrev*, Moe Nasr, Hayden Segboer, Crispin Corte, Ken Liu. AW Morrow Gastroenterology and Liver Centre, Royal Prince Alfred Hospital, Australia

10.1136/gutjnl-2020-IDDF.102
Background Frailty has been described in young patients with chronic inflammatory conditions. We aimed to determine the prevalence and impact of frailty among inflammatory bowel disease (IBD) outpatients

Methods Patients were prospectively recruited from our tertiary IBD clinic Nov 2018-Nov 2019. Frailty was defined by Fried Frailty Index (FFI). Crohn's Disease Activity Index (CDAI) for Crohn's disease (CD) or partial Mayo score for ulcerative colitis (UC) and IBD unclassified (IBDU), IBD questionnaire (IBDQ), Patient Health Questionnaire-9 (PHQ-9) and Charlson comorbidity index (CCI) were collected.

Results Forty-one patients were recruited (63\% female, median age $32,22 \%$ UC, 71\% CD, 7\% IBDU). Five patients $(12 \%)$ were frail $(\mathrm{FFI} \geq 3)$ and $36(88 \%)$ were non-frail: 20 $(49 \%)$ pre-frail $(\mathrm{FFI}=1-2), 16(39 \%)$ robust $(\mathrm{FFI}=0)$. Frail patients were older (median age $54 \mathrm{vs.31}, P=0.03$ ) with longer duration of IBD (median 20vs.6 years, $P=0.05$ ) compared to non-frail. FFI correlated with CCI (Pearson r.32, $P=0.04)$. Mean CDAI and median partial Mayo scores were higher in frail/pre-frail patients with CD (224 vs.112, $P<0.01$ ) and UC/IBDU (3vs.0.5, $P=0.03$ ) compared to robust patients. Conversely, the proportion of frail/pre-frail patients increased with IBD activity: remission (24\%), mild (36\%), moderate (89\%) and severe disease activity (100\%), $P P=0.02)$. Compared to robust patients, frail/pre-frail patients had higher mean PHQ-9 scores (11.0vs.7.1, $P=0.02)$ and higher depression rates (60\%vs.25\% with PHQ-9 $\geq 10$, $P=0.03)$. Anti-depressant use increased with FFI score $(11 \%$ $\mathrm{FFI}=0,17 \% \mathrm{FFI}=1,40 \% \mathrm{FFI}=2,100 \% \mathrm{FFI} \geq 3, P=0.02)$. Frail/pre-frail patients had lower mean IBDQ scores (141vs.169, $P=0.02$ ) compared to robust patients denoting worse quality of life (QOL).

Conclusions Frailty or prefrailty is common among IBD outpatients and associated with older age, comorbidity, IBD duration, disease activity, prednisone use, depression and lower QOL.

\section{IDDF2020-ABS-0163 REDUCED SURVIVAL POST-ENDOSCOPY FOR UPPER GASTROINTESTINAL BLEEDING IN THE COVID-19 ERA IS A SECONDARY EFFECT OF THE RESPONSE TO THE GLOBAL PANDEMIC}

${ }^{1}$ Oliver Tavabie*, ${ }^{2}$ Jennie Clough, ${ }^{3}$ Jonathan Blackwell, ${ }^{4}$ Maria Bashyam, ${ }^{5}$ Harry Martin, ${ }^{6}$ Anet Soubieres, ${ }^{6}$ Natalie Direkze, ${ }^{5}$ David Graham, ${ }^{3}$ Christopher Groves, ${ }^{7}$ Sean Preston, ${ }^{2}$ Sabina DeMartino, ${ }^{7}$ Upkar Gill, ${ }^{1}$ Bu'Hussain Hayee, ${ }^{1}$ Deepak Joshi. ${ }^{1}$ King's College Hospital, UK; ${ }^{2}$ Guy's and St Thomas' Hospital, UK; ${ }^{3}$ St George's Hospital, UK; ${ }^{4}$ St Mary's Hospital, UK; ${ }^{5}$ University College Hospital, UK; ${ }^{6}$ Imperial College Healthcare NHS Trust, UK; ${ }^{7}$ Royal London Hospital, UK

\subsection{6/gutjnl-2020-IDDF.103}

Background The COVID-19 pandemic has placed increased strain on healthcare systems worldwide with enormous reorganisation undertaken to support 'COVID-centric' services. Non-COVID-19 admissions have been shown to have reduced due to public health measures to halt viral transmission. We aimed to understand the impact of the response to the COVID-19 pandemic on the outcomes of upper gastrointestinal bleeds (UGI).

Methods A pan-London retrospective observational multicentre study comparing outcomes following endoscopy for UGI bleeds from 24th March 2020 to 20th April 2020 to the 


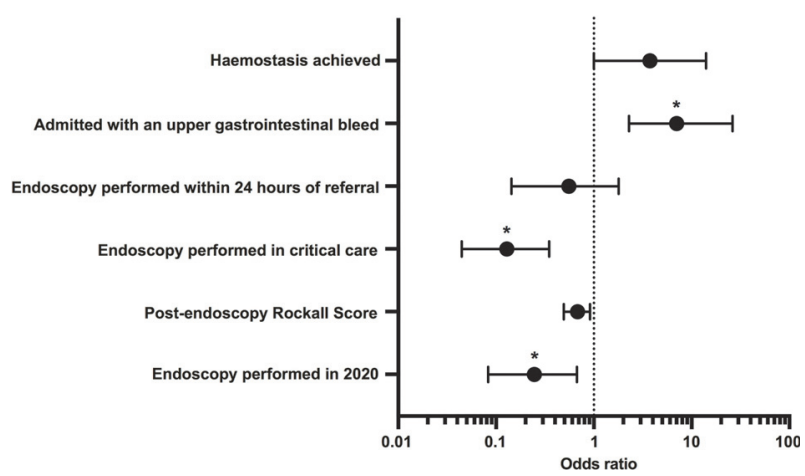

Abstract IDDF2020-ABS-0163 Figure 1 Odds ratio plot demonstrating outcomes of a multiple logistic regression model evaluating risk factors affecting 30-day survival post-endoscopy for UGI bleeds

corresponding dates in 2019. Primary outcome was in-hospital survival at 30 days with secondary outcomes of major rebleeding within 30 days post-procedure and intervention at time of endoscopy.

Results 224 endoscopies for 203 patients with UGI bleeds were included within this study. 19 patients were diagnosed with COVID-19. There was a $44.4 \%$ reduction in the number of procedures performed between 2019 and 2020. Endoscopies performed for UGI bleeds in the COVID-19 era were associated with an adjusted reduced 30-day survival (OR 0.25, 95\% CI 0.08-0.67) (figure 1). There was no increased risk of major re-bleeding or interventions during this era. Patients with COVID-19 did not have adjusted reduced survival or adjusted increased complication rates.

Conclusions Endoscopy for UGI bleeds in the COVID-19 era is associated with reduced survival. No clear cause has been identified but we suspect this is a secondary effect of the response to the COVID-19 pandemic. Urgent work is required to encourage the public to seek medical help if required and to optimise patient pathways to ensure that the best possible patient care is provided.

\section{IDDF2020-ABS-0164 CLINICAL SYMPTOMS, ENDOSCOPIC FINDINGS AND LOWER ESOPHAGEAL SPHINCTER PRESSURE IN PATIENTS WITH ABSENT CONTRACTILITY ON HIGH- RESOLUTION MANOMETRY}

${ }^{1}$ Hang Dao*, ${ }^{2}$ Hue Luu, ${ }^{2}$ Linh Nguyen. ${ }^{1}$ Hanoi Medical University, Vietnam; ${ }^{2}$ Institute of Gastroenterology and Hepatology, Vietnam

\subsection{6/gutjnl-2020-IDDF.104}

Background Absent contractility is a rare esophageal motility disorder, described mainly in patients with systemic diseases. There is no available data on the Asian population as well as in Vietnam. This study aims to describe clinical symptoms, upper gastrointestinal endoscopy findings (UGIE) and lower esophageal sphincter pressure (LES) in patients with absent contractility on high-resolution manometry (HRM).

Methods A cross-sectional study in 67 patients diagnosed with absent contractility on HRM at the Institute of Gastroenterology and Hepatology from March 2018 to April 2019.
Results During the study period, 2762 patients with upper gastrointestinal symptoms were performed HRM in which 67 patients $(2.4 \%)$ were diagnosed with absent contractility on HRM. There is no case with the past history of systemic diseases or diabetes. The female/male ratio was 1.6 , and the mean age was $43.2 \pm 14.8$. Common symptoms were regurgitation (74.6\%), heartburn (28.4\%) and dysphagia (32.8\%). $80.6 \%$ and $44.8 \%$ of the patients had an FSSG and GERDQ score $\geq 8$, respectively. On UGIE, 46\% had reflux esophagitis, mostly Los Angeles grade A. The prevalence of LES $<10$ $\mathrm{mmHg}$ and IRP4s $<5 \mathrm{mmHg}$ were significantly higher in patients with esophagitis on endoscopy.

Conclusions Clinical symptoms of patients with absent contractility were various and nonspecific. The prevalence of low LES pressure was significantly higher in patients having reflux esophagitis.

\section{IDDF2020-ABS-0167 ANXIETY AND DEPRESSION AMONG GASTROENTEROLOGISTS: AN ONLINE SURVEY IN BANGLADESH}

${ }^{1}$ Abdul Mumit Sarkar*, ${ }^{2}$ Madhusudan Saha, ${ }^{3}$ Mushtaque Ahmed Rana, ${ }^{4}$ Shasanka Kumar Saha. 'Rajshahi Medical College, Rajshahi, Bangladesh; ${ }^{2}$ North East Medical College, Sylhet, Bangladesh; ${ }^{3}$ Bangladesh Medical College, Dhaka, Bangladesh; ${ }^{4} \mathrm{M}$ Abdur Rahim Medical College, Dinajpur, Bangladesh

\subsection{6/gutjnl-2020-IDDF.105}

Background Anxiety and depression negatively affect physicians' performance leading to make errors in service providing. Moreover, there is a significant association between professional burnout and anxiety and depression. As a part of this doctor community, gastroenterologists are also at risk of suffering from mental health problems. This study was designed to see the prevalence of anxiety and depression among gastroenterologists in Bangladesh.

Methods Anxiety and depression negatively affect physicians' performance leading to make errors in service providing. Moreover, there is a significant association between professional burnout and anxiety and depression. As a part of this doctor community, gastroenterologists are also at risk of suffering from mental health problems. This study was designed to see the prevalence of anxiety and depression among gastroenterologist in Bangladesh.

Results Out of 166 gastroenterologists, 63 (37.95, mean age $20.2 \pm 8.0$ years, $96.8 \%$ female) responded. The service length of respondents varied from 5 years to 43 years (mean 20.29 \pm 8.09 ) and their working length as specialists varied from 1 to 38 years (mean $13.28 \pm 7.278$ ). In this series symptom scores of $13(20.6 \%)$ and $3(4.8 \%)$ respondents were consistent with borderline anxiety and overt anxiety respectively and symptom scores of $11(17.5 \%)$ and 2 (3.2\%) respondents were consistent with borderline and overt depression respectively. Among them, nine (14.2\%) respondents had both anxiety and depression (including borderline and overt cases). Anxiety was significantly more prevalent (9, 31.0\%) among gastroenterologists of 41-50 years age group $(p=0.007)$ and those are working as specialists less than or equal to five years $(6 ; 75 \%),[\mathrm{P}=0.014]$. Depression was also more common among gastroenterologists of age $41-50$ years group $7(24.13 \%)$, doing government service 10 (19.60\%), service length $\leq 15$ years 5 (29.41), and working as specialist $\leq 10$ years 7 (31.82\%), but the difference was not significant. 